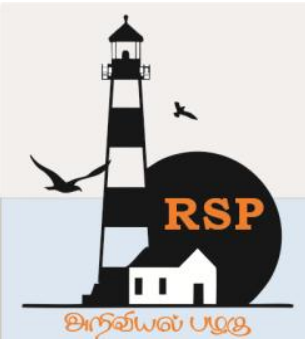

\title{
Strengthening WSN Applications in Oil and Gas Industries for Multiple Monitoring Frameworks
}

Mohammed Fasiuddin ${ }^{1}$, Dr Anupama A Deshpande ${ }^{2}$, Dr Mahabood Shaik ${ }^{3}$

${ }^{1}$ Research Scholar, Shri JJT University, Rajasthan

${ }^{2}$ Professor, Department of Electrical and Electronics Engineering, Shri JJT University, Rajasthan, India

${ }^{3}$ Assitant Professor, MJCET, Hyderabad, India.

fasiuddin2002@yahoo.com ${ }^{1}$

\section{Abstract}

The IEEE 802.15.4 technical standard has enabled low-power, low-cost and smart Wireless Sensor Networks (WSNs) capable of reliable multi-hop communications. The companies like International Oil and Gas Company (IOC), Shell Petroleum Development Company (SPDC) have become the first multinational Oil and Gas Companies operating in the Nigeria Niger Delta region switched from wired to wireless sensor technology. Thus, eliminated the need for cables thereby allowing data collection in remote, swampy areas and enabling new applications. However, using these Wireless Sensor Networks, there are concerns such as reliability, standardisation, energy consumption, data and physical security issues especially in the monitoring of oil and gas installations and infrastructures such as pipelines, oil wells, oil rigs and flow stations. Vandalising and sabotaging of oil pipelines and other infrastructure is common by oil thieves in a region even after the Wireless Sensor deployment. In this paper, the mechanisms that can be carried out to secure oil facilities and wireless sensors being attacked physically or remotely and report incidents on time are discussed.

\section{Keywords: Wireless Sensor Network, Data Distribution Node, Communication Relay Node.}

\section{Introduction}

A wireless sensor network (WSN) typically consists of a variety of inexpensive, low power, multifunctional sensor nodes that are placed at a desired location. WSN allows nodes to communicate wirelessly over short distances and organize into multi-hop mesh networks. WSNs can generally be described as networks of nodes that work together and can manage an environment that enables interactions between individuals or between a computer and its environment. A typical WSN sensor node consists of a wireless communication unit, a microprocessor, a data acquisition unit, a memory unit and a sensor board.
The microprocessor and memory unit maintain the frequency at which the sensor is measured or transmitted. The usefulness of WSN for environmental monitoring has been studied in the literature. For example, WSN was used to collect statistics on forest temperature and rainfall. In this document, we use a network of wireless sensors that uses a real-time environmental monitoring machine to successfully measure temperature, greenhouse gas concentration with $\mathrm{CO}, \mathrm{CO} 2$, and $\mathrm{CH} 4$ levels. The network consists of two nodes and a base station. Mainly based on IEEE 802.15.4, the ZigBee Wireless Module is used as the data exchange component of the sensor node. ZigBee has been 
selected as the cheapest and lowest power wireless conversation protocol and has proven to be a practical science for tactile applications. These radio modules are easy to configure, ideal for pointto-point and multipoint communication, and can be converted into mesh networks.[1-4]

The Wireless Sensor Community (WSN) is one of the world's most advanced technologies with features such as environmental monitoring, smart locations, scientific systems, robot detection, and military surveillance. The fact that so many countries depend on oil and gas is interested in the welfare, improvement and social balance of this proposal. As in Nigeria, hydrocarbon sources harbor the country's economic system, but given the turmoil in the Niger Delta, production and growth in the oil and gas sector are regularly limited. Nigeria's economic system is closely related to the oil sector, which, according to the World Bank, represents more than $95 \%$ of export earnings and around $85 \%$ of government revenue.

\section{Literature Review}

J. Yang and X. Li (2010) The rapid improvement and miniaturization of sensors, as well as the latest advances in communication technologies and wireless networks, allow scientists and engineers to strengthen the small sensor networks we use to continuously improve the physical health and balance of the environment in which we live. Can be used to display. The project provides a detailed study and implementation of WSNs for continuous, real-time environmental monitoring of greenhouse gases. To measure the concentration levels of various greenhouse gases, the tree topology WSN with two sensor nodes and a base station has been successfully built and tested using low-cost open source equipment. strictly. The sensor nodes were packed with carbon monoxide sensors, carbon dioxide sensors, methane sensors, temperature sensors, GPS modules, and a ZigBee wireless transmitter. A GPS module has been added to provide information about the location of the sensor. The base stations have an Arduino Uno microcontroller and a ZigBee receiver that can collect data from various sensors and send it to a good base station where the data can be stored and processed. A website has been developed where the captured data can be continuously monitored and displayed in real time. .[5-6]

N. Giannopoulos, C. Giannopoulos, A. Kameas (2009) Wireless sensor networks (WSN) is tending toward turning into a complete solution in communication protocols, embedded structures and low-power implementations. However, the resource constraints which includes, confined verbal exchange range, restricted energy, limited computing power, restrained bandwidth and the worry of intruders have limited the WSN applications. Since lightweight computational nodes that arecurrently being used in WSN pose precise project for many safety applications, the total lookup therefore, is the investigation of new safety strategies and gorgeous implementation for WSN nodes, which include a range of trade-offs such as implementation complexity, energy dissipation, safety flexibility and scalability. The intention of this research is to strengthen a community that has environment friendly and bendy key distribution scheme secured ample to stop algorithmic complexity and denial of service attacksas nicely as the community in a position to preserve energy. A review of previous research to date in the place of protection for WSNs used to be carried out and proposals are made based totally on safety schemes that gather statistics inan energyefficient mechanism via secured pre-allocation of keys, quicker clustering routing algorithm and dynamic based totally rekeying implementation.

F. Cuomo S. Della Luna, U. Monaco, U, and T. Melodia (2007) The monitoring of leaks in pipelines is an necessary issue to be addressed through researchers and the public. This is due the reality that they can have a superb have an impact on both economically and environmentally. In current years, the impact of leakages of pipelines carrying oil, gasoline and nuclear fluids have posed a threat on humans as nicely as marine life. This paper presents a survey of current techniques of detecting pipeline leaks with exceptional focal point on software program primarily based methods. These methods consist of bad stress wave, mass/volume balance, stress factor analysis, actual time transient modeling, statistical methods as well as techniques that employing digital signal processing. This paper also surveys some of the 


\section{www.rspsciencehub.com}

current lookup tries that center of attention on the employment of wi-fi sensor networks for leak detection and current research challenges that can be encountered in such environments.

\section{Methodology}

Communication reliability is one of the main reliability of using wireless sensor networks for pipeline monitoring and protection. Applications dedicated to pipeline devices need a network. Networks are usually extended by pipelines to collect internal measurements such as pressure, flow, temperature, area monitoring, pipeline protection cameras, pipeline furnace detection and various distribution sensors scattered across the pipeline. Collects and transfers external measurements such as pipeline leaks. Wireless sensor networks are less susceptible to these disruptions, but wired networks are very unreliable because the failure of the wire in the network disrupts the entire surveillance and security system. I do. For example, the image pipeline is monitored by the wireless sensor network. Each node in the diagram represents the Communication Relay Node $(\mathrm{CRN})$. Other underlying sensor nodes near the CRN node collect and filter information. Inspect the Data Distribution Node (DDN) until sensitive data is transferred from one communication relay node to another until it is transferred to another pipeline control center.

Reaching the node to the right and to the left is the wireless signal field for each node, as shown in the figure. The main problem with this model is that if there is a problem with the node in the network, the connection of the nodes is lost and the network is broken. Using a limited radio range reduces power consumption at nodes. However, there may also be a connectivity problem. In other words, you can disable the entire monitoring and security device for a single node pipeline. Large signal ranges can be used between sensor nodes. It uses additional power from the sensor battery, but provides more reliability if some nodes have a problem.

For example, each node in the diagram can communicate with two nodes on the left and two nodes on the right. For example, if node 3 and 5 are damaged, node 4 can still send detected data through node 2 or 6 . Increasing range increases reliability,
Volume 02 Issue 06 June 2020

but consumes more energy. Communication relay node. An example of this configuration is illustrated in the figure. In this network, nodes 3 and 5 fail. As a result, the radio range of node 4 has been expanded to reach nodes 2 and 6.

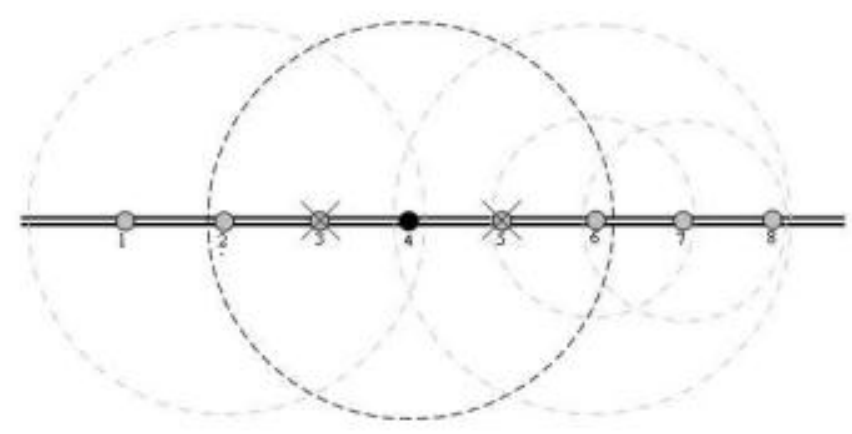

Fig.1. Automatic wireless range configuring Nodes can have different wireless range to stay connected

\section{[1]. Results}

The receiving node communicates with the local network. The environmental data provided by the sensor nodes is graphically displayed on the website. This website is integrated with HTML code, PHP server script and MySQL database tools. The website is designed so that users can choose between two sensors, and the drop-down option allows users to switch between greenhouse gases such as $\mathrm{CO} 2, \mathrm{CO}$ and $\mathrm{CH} 4$.

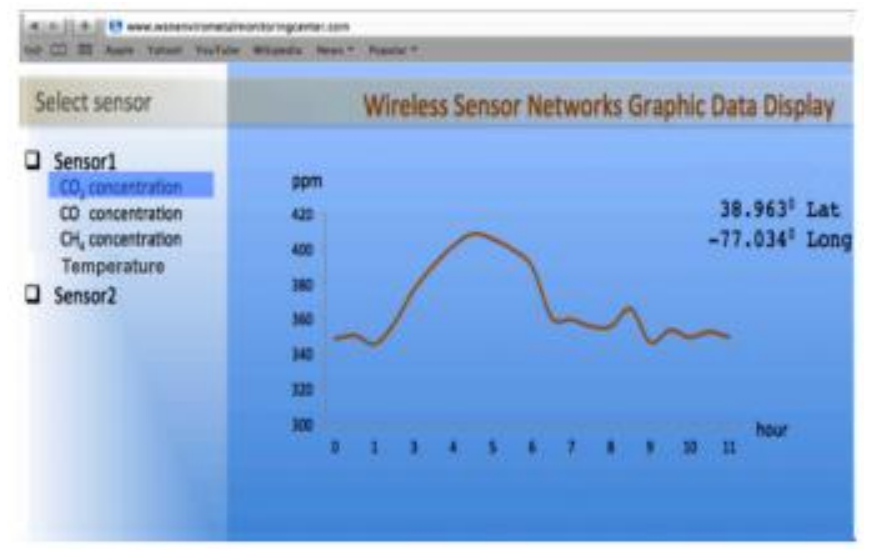

Fig.2.Graphic display of sensor data on a website

As the graph shows, the $\mathrm{x}$-axis shows the time it takes to take more than 12 hours every 30 minutes, and the y-axis shows the $\mathrm{CO} 2$ levels (ppm). When updating the website, real-time updates are available. The graph will be published on the 
website based on the data collected and disseminated in the database.

\section{Conclusion}

Security research on the host of wireless sensors is expanding. Creating a security protocol can be quite complex and requires a lot of skill. Protocols and routes should be well adapted, flexible, meet the requirements for reducing energy consumption, acceptable in practice and understandable in the real world of sensors. Therefore, it has been noted that regardless of the resource constraints in WSN, it is possible to obtain a WSN Protection Scheme that provides complete protection through energy efficient data collection, 3D visionization method, when detected. Acting inequality in society and in the future. The use of WSN cellular nodes in any environment, including the oil and gas industry, where pipeline breakdowns are common. When properly managed and implemented, these schemes can significantly change the scope of WSN and increase its usefulness in the industry.

\section{References}

\section{Journals}

[1].S. Hadim, J. Al-Jaroodi, and N. Mohamed.Trends in middleware for mobile ad hoc networks. The Journal of Communications, 1(4):11-21, July 2006.

[2].S. Hadim and N. Mohamed. Middleware challenges and approaches for wireless sensor networks. IEEE Distributed Systems, 7(3), March 2006.

[3].I. Jawhar and J. Wu. Qos support in tdmabased mobile ad hoc networks. The Journal of Computer Science and Technology (JCST), 20(6):797- 910, November 2005 Dekker, C. (1999). Carbon Nanotubes as Molecular Quantum Wires. Physics Today, 52(5), 22.

[4].I. Jawhar and J. Wu. Quality of sevice routing in mobile ad hoc networks.Resource Management in Wireless Networking, M. Cardei, I. Cardei, and D. -Z. Du (eds.), Springer, Network
Theory and Applications, 16:365- 400, 2005.

\section{Conference Proceedings}

[5]. Mittal. Ruchi and Bhatia. M.P.S "Wireless Sensor Networks for Monitoring the Environmental Activities" Computational Intelligence and Computing Research (ICCIC), IEEE International Conference, Coimbatore, India, December 2010, pp.1-5.

[6].K. Lu, Y. Qian, D. Rodriguez, W. Rivera, and M. Rodriguez "Wireless Sensor Networks for Environmental Monitoring Applications: A Design Framework", in Proc. IEEE Global Communications Conference, Washington, DC, November 2007, pp. 1108-1112. 